\title{
Uncertainty Aversion with Second-Order Utilities and Probabilities
}

\author{
Robert F. Nau \\ Fuqua School of Business, Duke University, Durham, North Carolina 27708-0120, robert.nau@duke.edu
}

\begin{abstract}
Subjective expected utility theory does not distinguish between attitudes toward uncertainty (ambiguous $S$ probabilities) and attitudes toward risk (unambiguous probabilities). Both are explained in terms of nonlinear utility for money rather than properties of events per se, hence, the decision maker displays the same attitude toward all sources of risk and uncertainty. There is ample evidence that real decision makers do not always behave (or even wish to behave) in this way, and instead they often distinguish between risk and uncertainty, as in Ellsberg's (1961) paradox. This paper presents a simple axiomatic model of nonneutral attitudes toward uncertainty and a behavioral test for uncertainty aversion that is applicable even if utility is state dependent. The decision maker may display different degrees of aversion toward gambles on different kinds of events, e.g., being systematically more averse toward gambles on events whose probabilities are more ambiguous. For such a decision maker, the elicitation of preferences among objective gambles may not yield the correct measure of risk aversion for modeling real-world decisions.
\end{abstract}

Key words: risk aversion; uncertainty aversion; ambiguity; state-dependent utility; Choquet expected utility; cumulative prospect theory; state-preference theory; Ellsberg's paradox; smooth preferences

History: Accepted by Detlof von Winterfeldt, decision analysis; received December 12, 2003. This paper was with the author 6 months for 1 revision.

\section{Introduction}

In the theory of subjective expected utility (SEU) (Savage 1954), there is no important distinction between objective and subjective probability: to a decision maker all events are qualitatively alike, differing only in their quantitative degrees of belief represented by probabilities that are always precise even in the absence of historical frequency data, symmetry arguments, or expert knowledge. Attitudes toward risk and uncertainty are explained as if they arise from nonlinear value for money, represented by a Bernoulli utility function, rather than properties of events per se. This idea has been enormously fruitful for modeling decisions and markets under uncertainty, but it has also remained somewhat controversial on both normative and descriptive grounds.

One of the most persistent challenges to the theory has been the phenomenon of "uncertainty aversion" (also called "ambiguity aversion") demonstrated by Ellsberg (1961), in which decision makers are typically more averse to betting on events whose probabilities are subjective and ambiguous than they are to betting on events whose probabilities are objective and unambiguous. This pattern of behavior violates a key assumption of Savage's (1954) theory, the coordinate independence axiom (P2) - otherwise known as the sure-thing principle-which implies that the utility of an act is an additively separable function of the consequences it yields in different states of the world. This axiom rules out complementarities between consequences received in mutually exclusive events, which in turn rules out any source dependence of attitudes toward risk and uncertainty. If coordinate independence is satisfied, risk attitudes may still be state dependent, but not dependent on how states are grouped together to form events on which bets are placed: the risk premia of small gambles must be additively separable across states. However, Savage's axioms also include an additional postulate (P3) that forces preferences to be state independent as well as source independent. These two independence postulates justify the conventional decision-analytic practice of assessing a decision maker's risk tolerance by asking her to evaluate casino gambles with objective probabilities and later using that same risk tolerance to guide decisions with respect to other, possibly more ambiguous sources of risk and uncertainty (e.g., the stock market, risks to life and health, etc.).

This paper develops an axiomatic model of choice under uncertainty that permits the decision maker's preferences to display both source dependence and state dependence, together with a behavioral test for uncertainty aversion that extends Ellsberg's experiment to conditions of state-dependent utility and unobservable prior stakes in events. Under this model, the decision maker may behave like an expected utility 
(EU) maximizer toward casino gambles, and she may also behave like an EU maximizer toward investments in the stock market, but she could be less risk averse toward the former than toward the latter (or perhaps vice versa), and the price she is willing to pay for a financial asset may include a measurable "uncertainty premium" as well as a risk premium.

Numerous other extensions of SEU theory have been proposed to accommodate uncertainty aversion, including Schmeidler's (1989) Choquet expected utility, Gilboa and Schmeidler's (1989) maxmin expected utility, Segal's (1987) application of anticipated utility, Tversky and Kahneman's (1992) cumulative prospect theory (axiomatized by Tversky and Wakker 1993), and a number of more recent models (Epstein 1999; Epstein and Zhang 2001; Ghirardato and Marinacci 2001, 2002; Klibanoff et al. 2005; Ergin and Gul 2004; Chew and Sagi 2003; Maccheroni et al. 2004). All of these uncertainty models relax the coordinate independence axiom to some extent-e.g., by restricting the set of acts to which it applies-but most of them still strive (like SEU) to represent the decision maker's preferences in terms of separate measures of belief and value, and in this regard they generally assume that preferences are state independent, along the lines of Savage's (1954) P3 or the similar monotonicity-inthe-prizes axiom of Anscombe and Aumann (1963). State-independent preferences are essential for constructing the constant acts that serve as important benchmarks in SEU theory as well as in many characterizations of aversion to risk and uncertainty. For example, Epstein and Zhang (2001, p. 275) remark: "The necessity of this axiom in our approach implies, in particular, that we have nothing to say about the meaning of ambiguity when preferences are state dependent." Beliefs with respect to ambiguous events are usually represented by nonadditive or set-valued probabilities, or other generalized belief measures, while values (which determine risk attitudes toward unambiguous events) are still represented by a stateindependent Bernoulli utility function. However, the state independence of preferences and the separability of beliefs and values are also controversial topics in SEU theory (e.g., Aumann 1971, Karni et al. 1983, Shafer 1986, Schervish et al. 1990, Karni and Mongin 2000, Nau 2001), and it is therefore of interest to try to model uncertainty aversion in such a way that the whole enterprise does not hinge on them.

In a recent companion paper (Nau 2003), it is shown how risk aversion can be measured and risky projects can be valued without assuming state independence and without separating probabilities from utilities, starting from the Arrow-Debreu statepreference approach to modeling choice under uncertainty in which acts are vectors of state-contingent wealth, rather than from the approaches of Savage
(1954) or Anscombe and Aumann (1963) in which acts are more abstract (and often imaginary) mappings of states to consequences or to objective lotteries over consequences. The aim of this paper is to show how uncertainty aversion can be modeled within the same framework. Whereas uncertainty aversion is commonly defined as convexity of preferences with respect to objective probabilistic mixtures of Anscombe-Aumann acts (Schmeidler 1989, Gilboa and Schmeidler 1989), or else as a comparative relation on preference orders that uses probabilistically sophisticated preferences and/or constant acts as benchmarks (Epstein 1999, Ghirardato and Marinacci 2002), uncertainty aversion is defined and measured here in terms of the nonseparability of risk premia for small gambles, without reference to objective or subjective probability or constant acts. However, risk-neutral probabilities (marginal betting rates) play a central role, as they do in markets under uncertainty.

A specific representation of smooth uncertaintyaverse preferences is derived from a nested, or twostage, independence assumption analogous to the axioms introduced by Kreps and Porteus (1979) for modeling attitudes toward the timing of resolution of uncertainty. The general form of the representation involves first- and second-order evaluation functions that do not separate probability from utility. Under stronger assumptions, the evaluation functions can be formally (if not necessarily epistemically) decomposed into first- and second-order Bernoulli utility functions together with unique marginal and conditional probabilities, hence, the model gives rise to second-order utilities and probabilities (SOUP). The second-order evaluation or utility functions may amplify the degree of risk aversion toward more ambiguous events (for example, states of nature as opposed to artificially randomized events) permitting the decision maker to display different attitudes toward different sources of risk and uncertainty.

Structurally similar two-stage models of smooth uncertainty-averse preferences have also been developed recently, and independently, by Ergin and Gul (2004), Klibanoff et al. (2005), and Chew and Sagi (2003), using the Savage (1954) framework. Ergin and Gul's model posits two sets of "issues," $a$ and $b$, which are analogous to the sets $\mathscr{A}$ and $\mathscr{B}$ in this paper, and uses nested versions of Savage's P4 (comparative probability axiom) to obtain a notion of second-order probabilistic sophistication as well as second-order utility. Klibanoff et al.'s model explicitly involves second-order beliefs revealed by preferences for second-order acts, and the second-order utility function captures aversion to the second-order uncertainty. Gilboa and Schmeidler's (1989) maxmin 
EU model and the more general variational-preference model of Maccheroni et al. (2004), which use the Anscombe-Aumann (1963) framework, also potentially allow for uncertainty aversion with smooth preferences. In contrast, the Choquet expected utility (CEU) model of Schmeidler (1989) and the cumulative prospect theory (CPT) model of Tversky and Kahneman (1992) feature a utility function that is nondifferentiable, so that the decision maker displays local uncertainty aversion only when her status quo wealth happens to lie on a boundary between comonotonic sets, where her indifference curves are kinked.

This paper is organized as follows. Section 2 introduces the modeling framework and basic riskaversion concepts for decision makers with smooth preferences and inseparable probabilities and utilities, following Nau (2003). Section 3 defines uncertainty aversion in the same terms and gives simple examples of utility functions that rationalize Ellsberg's singleurn and two-urn paradoxes. Section 4 presents two representation theorems for the SOUP model, based on nested independence conditions for ambiguous and unambiguous events. Section 5 presents concluding comments. Proofs are given in the original working version of the paper and are available on request.

\section{Modeling Framework and Risk-Aversion Concepts}

The modeling framework used throughout this paper is that of state-preference theory (Arrow 1964, Debreu 1959, Hirshleifer 1965), which encompasses both EU and non-EU models of choice under uncertainty. An act in this framework is a mapping from observable states of the world to quantities of a single consumption good (money). Assume that the state space can be represented as a Cartesian product, $\mathscr{A} \times \mathscr{B}$, where $\mathscr{A}=\left\{A_{1}, \ldots, A_{J}\right\}$ and $\mathscr{B}=\left\{B_{1}, \ldots, B_{K}\right\}$ are finite sets, with $J, K \geq 2$. An act will be denoted by a doubly-subscripted vector $\mathbf{w}=\left(w_{11}, w_{12}, \ldots, w_{I K}\right)$, where $w_{j k} \in \Re$ is the payoff in state $A_{j} B_{k}$. The sets $\mathscr{A}$ and $\mathscr{B}$ are interpreted to represent two logically independent sources of risk and uncertainty, and their subsets will be referred to as $\mathscr{A}$-events and $\mathscr{B}$-events, respectively. It is assumed that the $\mathscr{A}$-events are known to be regarded by the decision maker as "more ambiguous" than the $\mathscr{B}$-events, merely to provide a directional arrow for characterizing her attitude toward uncertainty: a systematic preference for betting on $\mathscr{B}$-events rather than $\mathscr{A}$-events will be used below to define aversion rather than attraction to uncertainty. To fix ideas, it might be supposed that elements of $\mathscr{B}$ are states of an objective randomization device while elements of $\mathscr{A}$ are states of the natural world, as in the "horse lotteries" that are the objects of choice in Anscombe and Aumann's (1963) axiomatization of SEU, as well as in Choquet and maxmin EU and related models. However, as shown by Debreu (1960), it is not necessary that $\mathscr{B}$-events have objective probabilities nor that all decision makers regard the same events as more ambiguous. The property of "ambiguity" is viewed here as a psychological primitive that helps to categorize observed patterns of source-dependent risk attitudes, but it could have many underlying meanings: the events that a decision maker considers more ambiguous could be those that are further from her interests, experience, or competence as a risk taker, not merely those that lack objective data.

Assume that the decision maker's preferences among acts are described by a binary relation $\succcurlyeq$ that satisfies the usual axioms of consumer theory (completeness, transitivity, continuity, monotonicity, etc.) leading to a smooth, ordinal utility representation, summarized as:

Axiom 1. The decision maker's preference relation $\succcurlyeq$ is represented by an ordinal utility function $U(\mathbf{w})$ that is everywhere twice differentiable with a strictly positive gradient.

The normalized gradient of $U$ evaluated at $\mathbf{w}$ is a probability distribution $\pi(\mathbf{w})$, whose $j k$ th element is

$$
\pi_{j k}(\mathbf{w})=\frac{\partial U(\mathbf{w}) / \partial w_{j k}}{\sum_{m=1}^{J} \sum_{n=1}^{K} \partial U(\mathbf{w}) / \partial w_{m n}} .
$$

$\pi_{j k}(\mathbf{w})$ is the rate at which the decision maker would indifferently bet infinitesimal amounts of money on or against the occurrence of state $A_{j} B_{k}$ when in possession of act $\mathbf{w}$. The distribution $\boldsymbol{\pi}(\mathbf{w})$ is invariant to monotonic transformations of $U$ and is observable, regardless of whether the decision maker has beliefs representable by unique probabilities. It is commonly known as a risk-neutral probability distribution because the decision maker prices very small assets in a seemingly risk-neutral manner with respect to it. A decision maker's risk-neutral probabilities at act $\mathbf{w}$ are implicitly a function of her beliefs, her attitudes toward risk and uncertainty, and her prior stakes in events, although their effects are typically confounded.

The risk-neutral distribution $\boldsymbol{\pi}(\mathbf{w})$ determines the first-order properties of the decision maker's local preferences in the vicinity of $\mathbf{w}$. Second-order properties of local preferences (risk premia, etc.) are determined by the matrix $\mathbf{R}(\mathbf{w})$, whose element in row $j k$ and column $m n$ is

$$
r_{j k, m n}(\mathbf{w})=-\frac{\partial^{2} U(\mathbf{w}) / \partial w_{j k} \partial w_{m n}}{\partial U(\mathbf{w}) / \partial w_{j k}} .
$$


$\mathbf{R}(\mathbf{w})$ generalizes the familiar Pratt-Arrow measure to the present setting and is appropriately called the riskaversion matrix (Nau 2003). The risk-aversion matrix is closely related to the matrix of derivatives of the riskneutral probabilities and is a measure of the relative curvature of the decision maker's indifference curves in the neighborhood of act $\mathbf{w}$.

If no additional restrictions are placed on preferences, the individual is economically rational in the sense that she cannot be exploited as a money pump, but she has non-EU preferences if her valuation of a risky asset is not decomposable into a product of probabilities for states and utilities for payoffs. For example, she may behave as if amounts of money received in some pairs of states are complementary goods, which is forbidden under EU theory. However, as shown by Debreu (1960), if her preferences also satisfy the coordinate independence axiom (Savage's P2, 1954), then $U(\mathbf{w})$ has the cardinal, additively separable form

$$
U(\mathbf{w})=\sum_{j=1}^{J} \sum_{k=1}^{K} v_{j k}\left(w_{j k}\right),
$$

which is unique up to positive affine transformations and can be interpreted as state-dependent EU without separation of probabilities from utilities. The functions $\left\{v_{j k}\right\}$ represent both beliefs and values, and are called evaluation functions. In this special case, the risk-neutral distribution satisfies $\pi_{j k}(\mathbf{w}) \propto v_{j k}^{\prime}\left(w_{j k}\right)$, and $\mathbf{R}(\mathbf{w})$ reduces to an observable diagonal matrix, whose $j k$ th diagonal element is $-v_{j k}^{\prime \prime}\left(w_{j k}\right) / v_{j k}^{\prime}\left(w_{j k}\right)$, a state-dependent Pratt-Arrow measure for state $A_{j} B_{k}$. If the evaluation functions $\left\{v_{j k}\right\}$ are positively linearly related, which is true if the decision maker satisfies a stronger axiom of trade-off consistency that combines P2 and P3 (Wakker 1989, Tversky and Wakker 1993), then $U(\mathbf{w})$ has the state-independent EU form

$$
U(\mathbf{w})=\sum_{j=1}^{J} \sum_{k=1}^{K} p_{j k} u\left(w_{j k}\right)
$$

where $\mathbf{p}$ is a unique probability distribution and $u(w)$ is a state-independent Bernoulli utility function that is unique up to positive affine transformations, as in Savage's (1954) model. In this case, $\pi_{j k}(\mathbf{w}) \propto p_{j k} u^{\prime}\left(w_{j k}\right)$, i.e., the risk-neutral distribution is merely the product of probabilities and relative marginal utilities for money, and the $j k$ th diagonal element of $\mathbf{R}(\mathbf{w})$ is $-u^{\prime \prime}\left(w_{j k}\right) / u^{\prime}\left(w_{j k}\right)$, the usual state-independent PrattArrow measure evaluated at wealth $w_{j k}$.

Following Yaari (1969) and Nau (2003), the decision maker is defined to be (weakly) risk averse if her preferences are convex with respect to mixtures of payoffs, which means that $\mathbf{w} \succcurlyeq \mathbf{x}$ implies $\alpha \mathbf{w}+(1-\alpha) \mathbf{x} \succcurlyeq \mathbf{x}$ for all $\alpha \in(0,1)$, where $\alpha \mathbf{w}+(1-\alpha) \mathbf{x}$ denotes the act whose payoff in state $A_{j} B_{k}$ is $\alpha w_{j k}+(1-\alpha) x_{j k}$. Payoff convexity of preferences holds if and only if the ordinal utility function $U$ is quasi-concave. The decision maker's degree of local risk aversion can be measured by the difference between her risk-neutral expected value of an asset and the price at which she is actually willing to buy it. Let $\mathbf{z}$ denote the payoff vector of a risky asset such as a bet. The decision maker's buying price for $\mathbf{z}$ when already in possession of act $\mathbf{w}$, denoted $B(\mathbf{z} ; \mathbf{w})$, is determined by

$$
U(\mathbf{w}+\mathbf{z}-B(\mathbf{z} ; \mathbf{w}))=U(\mathbf{w}) .
$$

The (buying) risk premium associated with $\mathbf{z}$ at act $\mathbf{w}$, here denoted $b(\mathbf{z} ; \mathbf{w})$, is the difference between the asset's risk-neutral expected value and its buying price:

$$
b(\mathbf{z} ; \mathbf{w})=E_{\pi(\mathbf{w})}[\mathbf{z}]-B(\mathbf{z} ; \mathbf{w}) .
$$

It follows (as a property of quasi-concavity) that the decision maker is risk averse if and only if her risk premium is nonnegative for every asset $\mathbf{z}$ at every act $\mathbf{w}$. If $\mathbf{z}$ is a neutral asset $\left(E_{\boldsymbol{\pi}(\mathbf{w})}[\mathbf{z}]=0\right)$, its risk premium has the following second-order approximation (Nau 2003) that generalizes Pratt's (1964) formula:

$$
b(\mathbf{z} ; \mathbf{w}) \approx \frac{1}{2} \mathbf{z}^{\mathrm{T}} \mathbf{\Pi}(\mathbf{w}) \mathbf{R}(\mathbf{w}) \mathbf{z},
$$

where $\mathbf{R}(\mathbf{w})$ is the risk-aversion matrix and $\Pi(\mathbf{\Pi})=$ $\operatorname{diag}(\boldsymbol{\pi}(\mathbf{w}))$.

In the special case where $U$ is additively separable, the risk-premium approximation formula reduces to

$$
b(\mathbf{z} ; \mathbf{w}) \approx \frac{1}{2} E_{\boldsymbol{\pi}(\mathbf{w})}\left[\mathbf{r}(\mathbf{w}) \mathbf{z}^{2}\right]=\frac{1}{2} \sum_{j=1}^{J} \sum_{k=1}^{K} \pi_{j k} r_{j k}\left(w_{j k}\right) z_{j k}^{2},
$$

where $r_{j k}\left(w_{j k}\right)=-v_{j k}^{\prime \prime}\left(w_{j k}\right) / v_{j k}^{\prime}\left(w_{j k}\right)$ is the Pratt-Arrow measure for state $A_{j} B_{k}$, as noted above. Hence, if the decision maker satisfies coordinate independence, her local preferences are completely described (up to second-order effects) by a pair of numbers for each state-a risk-neutral probability $\pi_{j k}(\mathbf{w})$ and a state-dependent risk-aversion measure $r_{j k}\left(w_{j k}\right)$ that are uniquely determined and observable-and her risk premium for a small asset is approximately onehalf the weighted, risk-neutral variance of its payoffs, with the state-dependent risk-aversion measures serving as the weights. If the evaluation functions $\left\{v_{j k}\right\}$ are concave, the decision maker is risk averse but uncertainty neutral because her risk premia are additively separable across states and thus insensitive to the way states are grouped together in the definitions of events.

\section{Definition and Examples of Uncertainty Aversion}

Uncertainty aversion in this setting arises as a special case of second-order risk aversion in which the risk 
premium of a gamble depends not only on the riskneutral variance of its payoffs, but also on the relative ambiguity of the events over which the variance occurs. Intuitively, a decision maker is averse to uncertainty if she prefers to bet on less ambiguous events, other things being equal. To make this notion precise, define an " $A$-spread" and a corresponding " $B$-spread" between any two logically independent events $A \subset \mathscr{A}$ and $B \subset \mathscr{B}$ in the following way. Let $\left\{\pi_{A B}, \pi_{A \bar{B}}, \pi_{\bar{A} B}, \pi_{\bar{A} \bar{B}}\right\}$ denote the local risk-neutral probabilities of the four possible joint realizations of $A$ and $B$, which (by Axiom 1) are all strictly positive. That is, $\pi_{A B}=\sum\left\{\pi_{j k}(\mathbf{w}) \mid A_{j} \in A, B_{j} \in B\right\}$, where $\mathbf{w}$ is status quo wealth. (These quantities are observable as the decision maker's marginal betting rates on the four events.) Let $\Delta$ denote a positive or negative quantity of money (just) large enough in magnitude so that some assets with payoffs comparable to $\Delta$ have risk premia that are noticeably different from zero. Then, an $A$-spread and its corresponding $B$-spread of size $\Delta$ are the neutral assets whose payoffs depend jointly on $A$ and $B$ (see Table 1).

Note that an $A$-spread is a bet on $A$ : the sign of the payoff depends only on whether $A$ occurs but the magnitude may also depend on whether $B$ occurs, because the payoff is inversely proportional to the risk-neutral probability of the joint event. Similarly, the corresponding $B$-spread is a bet on $B$ whose payoff magnitude may depend on $A$. The payoffs are scaled precisely so that the contribution to the riskneutral expected payoff is $\pm \Delta$ in every cell of the table. By construction, both bets are neutral (i.e., have zero risk-neutral expected value) and both must have the same risk premium for a decision maker whose preferences satisfy coordinate independence, because for such a person the risk premium is obtained (to a second-order approximation) by squaring the payoffs, multiplying them by the risk-neutral probabilities and risk-aversion measures of their respective states, and summing, as shown in Equation (1). A systematic difference in their risk premia therefore serves to reveal local violations of coordinate independence and to provide a benchmark for defining uncertainty aversion:

\begin{tabular}{lcc} 
Table 1 & $A$-Spreads and $B$-Spreads Defined \\
\hline & $A$-spread & $B$-spread \\
\hline$A B$ & $\frac{+\Delta}{\pi_{A B}}$ & $\frac{+\Delta}{\pi_{A B}}$ \\
$A \bar{B}$ & $\frac{+\Delta}{\pi_{A \bar{B}}}$ & $\frac{-\Delta}{\pi_{A \bar{B}}}$ \\
$\bar{A} B$ & $\frac{-\Delta}{\pi_{\bar{A} B}}$ & $\frac{+\Delta}{\pi_{\bar{A} B}}$ \\
$\bar{A} \bar{B}$ & $\frac{-\Delta}{\pi_{\bar{A} \bar{B}}}$ & $\frac{-\Delta}{\pi_{\bar{A} \bar{B}}}$ \\
\hline
\end{tabular}

Table 2 Ellsberg's Two-Urn Paradox

\begin{tabular}{lrrrr}
\hline & a & b & c & d \\
\hline Red I, Red II & 100 & 100 & -100 & -100 \\
Red I, Black II & 100 & -100 & -100 & 100 \\
Black I, Red II & -100 & 100 & 100 & -100 \\
Black I, Black II & -100 & -100 & 100 & 100 \\
\hline
\end{tabular}

Definition 1. The decision maker is locally uncertainty averse at act $\mathbf{w}$ if, for every $A$-event $A$ and $\mathscr{B}$-event $B$, there is some $\Lambda$ such that a $B$-spread of size $\Delta$ is strictly preferred to the corresponding $A$-spread for all $0<|\Delta|<\Lambda$. The decision maker is uncertainty averse if she is locally uncertainty averse at all $\mathbf{w}$.

The classic demonstration of uncertainty aversion is Ellsberg's (1961) two-urn paradox, in which urn I contains 100 balls that are red and black in unknown (ambiguous) proportions, while urn II contains exactly 50 red balls and 50 black balls. A single ball is to be randomly drawn from each urn, and the subject is asked to state her preferences among pairs of gambles, where amounts in the cells are payoffs received according to the colors drawn (see Table 2).

The typical response pattern is $\mathbf{b} \succ \mathbf{a}$ and $\mathbf{d} \succ \mathbf{c}$, i.e., the subject strictly prefers to bet on the color of the ball drawn from the less ambiguous urn, regardless of whether the winning color is red or black, which is a direct violation of coordinate independence. This experiment exactly fits the framework of Definition 1 : Let $A_{1}\left[A_{2}\right]$ denote the event that the ball drawn from urn I is red [black], and let $B_{1}\left[B_{2}\right]$ denote the event that the ball drawn from urn II is red [black], so that the relevant state space is $\left\{A_{1} B_{1}, A_{1} B_{2}, A_{2} B_{1}, A_{2} B_{2}\right\}$. If the subject has no prior stakes in the experiment and no reason to consider one color in urn I to be more likely than the other, then her risk-neutral probabilities should be equal to $1 / 4$ for all four outcomes, in which case a and $\mathbf{c}$ are $A_{1}$-spreads of size $\Delta=25$ and $\Delta=-25$, respectively, while $\mathbf{b}$ and $\mathbf{d}$ are $B_{1}$-spreads of size $\Delta=25$ and $\Delta=-25$, respectively, and the typical subject prefers the $B_{1}$-spread over the $A_{1}$-spread regardless of the sign of $\Delta$. Under more general conditions, $A$-spread and $B$-spread bets allow the magnitudes of the payoffs to vary inversely with the local risk-neutral probabilities to compensate for asymmetries in events, stochastic prior wealth, and/or statedependent utility.

It is straightforward to construct a smooth utility function that exhibits uncertainty aversion in the twourn paradox, such as the following:

$$
\begin{aligned}
U(\mathbf{w})= & -\frac{1}{2} \exp \left(-\alpha\left(\frac{1}{2} w_{11}+\frac{1}{2} w_{12}\right)\right) \\
& -\frac{1}{2} \exp \left(-\alpha\left(\frac{1}{2} w_{21}+\frac{1}{2} w_{22}\right)\right),
\end{aligned}
$$

where $\alpha>0$ and $w_{j k}$ as usual denotes the payoff in state $A_{j} B_{k}$. An axiomatic basis for a function 
of this type will be given in the next section, but briefly its logic is as follows. First, conditional on each of the ambiguous events $A_{1}$ and $A_{2}$, the decision maker evaluates the payoffs via a first-order EU calculation that represents her attitude toward pure risk, in this case with equal conditional probabilities assigned to $B_{1}$ and $B_{2}$ and linear first-order utility for money (i.e., risk neutrality). Second, the resulting "uncertainty profile" of conditional EUs is evaluated by performing a second-order EU calculation that represents her attitude toward uncertainty, in this case assuming equal marginal probabilities for $A_{1}$ and $A_{2}$ and using an exponential second-order utility function with parameter $\alpha$ (i.e., "constant absolute aversion to uncertainty"). The overall effect is to selectively depreciate acts whose uncertainty profiles exhibit more variance, and thus a (whose uncertainty profile is $\{100,-100\}$ ) is valued less than $\mathbf{b}$ (whose uncertainty profile is $\{0,0\}$ ), and likewise for $\mathbf{c}$ and b. (Interestingly, (2) can also be reinterpreted from the perspective of Klibanoff et al.'s (2005) model: it is as if the decision maker believes that the unknown red/black proportion is equally likely to be 0 or 1 , and she is averse to this second-order uncertainty.)

Applying the risk-aversion concepts of the preceding section to this example, the risk-aversion matrix is $\mathbf{R}(\mathbf{w}) \equiv \frac{1}{2} \alpha \mathbf{M}$, where $\mathbf{M}$ has a block structure with ones in its upper left and lower right $2 \times 2$ submatrices and zeroes elsewhere. The payoff vector of an $A_{1}$-spread is $\mathbf{z}=\left(+\Delta / \pi_{11},+\Delta / \pi_{12},-\Delta / \pi_{21}\right.$, $\left.-\Delta / \pi_{22}\right)$, while that of a $B_{1}$-spread is $\mathbf{z}=\left(+\Delta / \pi_{11}\right.$, $\left.-\Delta / \pi_{12},+\Delta / \pi_{21},-\Delta / \pi_{22}\right)$. Applying the formula $b(\mathbf{z} ; \mathbf{w}) \approx \frac{1}{2} \mathbf{z}^{\mathrm{T}} \boldsymbol{\Pi}(\mathbf{w}) \mathbf{R}(\mathbf{w}) \mathbf{z}$, the approximate risk premium for a $B_{1}$-spread is zero (in fact, it is exactly zero), while that of an $A_{1}$-spread is $\frac{1}{2} \alpha \Delta^{2} \sum_{j k} \pi_{j k}^{-1}>0$. If prior wealth is nonstochastic, then $\pi_{j k}=1 / 4$ for all $j k$, the payoffs are all $\pm 4 \Delta$, and the $A_{1}$-spread risk premium is approximately $\frac{1}{2} \alpha(4 \Delta)^{2}$. The subject is therefore risk neutral with respect to bets on urn II and risk averse with respect to bets on urn I: she behaves toward the latter as if she were an EU maximizer who believes that red and black are equally likely and whose PrattArrow risk-aversion measure is equal to $\alpha$.

In Ellsberg's (1961) single-urn paradox, the urn contains 30 red balls and 60 balls that are yellow and black in unknown proportions, from which a single ball will be randomly drawn, and the subject is asked to state her preferences within sets of gambles (see Table 3).

Table 3 Ellsberg's Single-Urn Paradox

\begin{tabular}{lrrrrrr}
\hline & a & b & c & d & e & f \\
\hline Red & 100 & 0 & 0 & 0 & 100 & 100 \\
Yellow & 0 & 100 & 0 & 100 & 0 & 100 \\
Black & 0 & 0 & 100 & 100 & 100 & 0 \\
\hline
\end{tabular}

The typical response pattern is $\mathbf{a} \succ \mathbf{b} \sim \mathbf{c}$ and $\mathbf{d} \succ$ $\mathbf{e} \sim \mathbf{f}$. That is, a subject prefers to stake a $\$ 100$ prize on the draw of a red ball rather than yellow or black, but if the prize is to be won when either of two specified colors is drawn, she prefers yellow or black over either red or yellow or red or black, which again is a direct violation of coordinate independence.

The single-urn experiment does not immediately fit into the framework of Definition 1, because there are only three payoff-relevant states. (It also requires the use of more costly and/or invasive nonneutral gambles, because one set of alternatives dominates the other.) However, if the story of the urn is taken literally, it is easy to refine the state space so that the ambiguous and unambiguous events are logically independent, as Definition 1 requires, by continuing to draw balls from the urn (without replacement) until a non-red ball is obtained. Let $A_{1}\left[A_{2}\right]$ denote the event that the first non-red ball is yellow [black], and let $B_{1}\left[B_{2}\right]$ denote the event that the first ball is red [not red]. Then, a $B_{1}$-spread is a bet on red versus not red as the color of the first ball, while the corresponding $A_{1}$-spread is a bet on yellow versus black as the color of the first non-red ball. For small $\Delta$, an uncertainty averse decision maker should prefer the former over the latter-even with stochastic prior wealth and/or state-dependent utility. In the simplest case where prior wealth is nonstochastic and yellow and black are regarded symmetrically by the decision maker, it is reasonable to suppose that the risk-neutral distribution over the four states $\left\{A_{1} B_{1}, A_{1} B_{2}, A_{2} B_{1}, A_{2} B_{2}\right\}$ is $\boldsymbol{\pi}(\mathbf{w})=(1 / 6,1 / 3,1 / 6,1 / 3)$, where the spread bets are as in Table 4 .

The $A_{1}$-spread is an even-odds bet on or against yellow as the color of the first non-red ball (with a doubling of the stake if the first ball is red), while the $B_{1}$-spread is a two-to-one bet on or against red as the color of the first ball. A strict preference for the $B_{1}$-spread, regardless of the sign of $\Delta$, can be explained by a utility function of the same general form as (2) but with different conditional probabilities for the $\mathscr{B}$-events:

$$
\begin{aligned}
U(\mathbf{w})= & -\frac{1}{2} \exp \left(-\alpha\left(\frac{1}{3} w_{11}+\frac{2}{3} w_{12}\right)\right) \\
& -\frac{1}{2} \exp \left(-\alpha\left(\frac{1}{3} w_{21}+\frac{2}{3} w_{22}\right)\right) .
\end{aligned}
$$

Table $4 \quad A$-Spreads and $B$-Spreads for the Refined Single-Urn Paradox

\begin{tabular}{lcc}
\hline & $A_{1}$-spread & $B_{1}$-spread \\
\hline$A_{1} B_{1}$ (Red first then yellow) & $+6 \Delta$ & $+6 \Delta$ \\
$A_{1} B_{2}$ (Yellow first) & $+3 \Delta$ & $-3 \Delta$ \\
$A_{2} B_{1}$ (Red first then black) & $-6 \Delta$ & $+6 \Delta$ \\
$A_{2} B_{2}$ (Black first) & $-3 \Delta$ & $-3 \Delta$ \\
\hline
\end{tabular}


A decision maker with this utility function and no prior stakes in the experiment will also exhibit the classic pattern $\mathbf{a} \succ \mathbf{b} \sim \mathbf{c}$ and $\mathbf{d} \succ \mathbf{e} \sim \mathbf{f}$ in Table 3 .

\section{Axiomatic Models of Second-Order Utility}

The examples in the preceding section illustrate how a risk-averse decision maker might behave like an EU maximizer with respect to bets on events having the same degree of ambiguity, while exhibiting higher degrees of risk aversion toward bets on events that are more ambiguous. This section presents axiomatic models of such behavior, yielding a general class of utility functions of which (2) and (3) are special cases. Insofar as coordinate independence forces uncertainty neutrality, it is necessary to weaken this axiom to allow for uncertainty aversion. The strategy followed here will be to modify the axiom so that it treats $\mathscr{A}$-events differently from $\mathscr{B}$-events. For any event $E$ and acts $\mathbf{w}$ and $\mathbf{x}$, let $\mathbf{w}_{E} \mathbf{x}_{-E}$ denote the act that agrees with $\mathbf{w}$ on $E$ and agrees with $\mathbf{x}$ on $\bar{E}$. Now suppose that preferences among acts satisfy the following partition-specific independence conditions:

Axiom 2 ( $A$-IndePendence). $\mathbf{w}_{A} \mathbf{x}_{-A} \succcurlyeq \mathbf{w}_{A}^{*} \mathbf{x}_{-A} \Leftrightarrow$ $\mathbf{w}_{A} \mathbf{x}_{-A}^{*} \succcurlyeq \mathbf{w}_{A}^{*} \mathbf{x}_{-A}^{*}$ for all acts $\mathbf{w}, \mathbf{w}^{*}, \mathbf{x}, \mathbf{x}^{*}$ and every A-event $A$. If $J=2$, then $\succcurlyeq$ also satisfies the corresponding hexagon condition: $\mathbf{w}_{A} \mathbf{x}_{-A} \sim \mathbf{w}_{A}^{*} \mathbf{x}_{-A}^{*}$ and $\mathbf{w}_{A} \mathbf{x}_{-A}^{* *} \sim \mathbf{w}_{A}^{*} \mathbf{x}_{-A}$ and $\mathbf{w}_{A}^{*} \mathbf{x}_{-A} \sim \mathbf{w}_{A}^{* *} \mathbf{x}_{-A}^{*} \Rightarrow \mathbf{w}_{A}^{*} \mathbf{x}_{-A}^{* *} \sim \mathbf{w}_{A}^{* * *} \mathbf{x}_{-A}$.

Definition 2 ( $\mathscr{A}$-Conditional Preference). For any $\mathscr{A}$-event $A, \mathbf{w} \succcurlyeq_{A} \mathbf{w}^{*}$ means that $\mathbf{w}_{A} \mathbf{x}_{-A} \succcurlyeq \mathbf{w}_{A}^{*} \mathbf{x}_{-A}$ for some $\mathbf{x}$, and $\succcurlyeq_{j}$ denotes the special case of $\succcurlyeq_{A}$ when $A=A_{j}$.

Axiom 3 (Conditional $\mathscr{B}$-IndePendence). $\mathbf{w}_{\mathbf{B}} \mathbf{x}_{-B} \succcurlyeq_{j}$ $\mathbf{w}_{B}^{*} \mathbf{x}_{-B} \Leftrightarrow \mathbf{w}_{B} \mathbf{x}_{-B}^{*} \succcurlyeq_{j} \mathbf{w}_{B}^{*} \mathbf{x}_{-B}^{*}$ for all acts $\mathbf{w}, \mathbf{w}^{*}, \mathbf{x}, \mathbf{x}^{*}$ and every $\mathscr{B}$-event $B$. If $K=2$, then $\succcurlyeq_{\text {; }}$ also satisfies the corresponding hexagon condition: $\mathbf{w}_{B} \mathbf{x}_{-B} \sim_{j} \mathbf{w}_{B}^{*} \mathbf{x}_{-B}^{*}$ and $\mathbf{w}_{B} \mathbf{x}_{-B}^{* *} \sim_{j} \mathbf{w}_{B}^{*} \mathbf{x}_{-B}$ and $\mathbf{w}_{B}^{*} \mathbf{x}_{-B} \sim_{j} \mathbf{w}_{B}^{* *} \mathbf{x}_{-B}^{*} \Rightarrow \mathbf{w}_{B}^{*} \mathbf{x}_{-B}^{* *} \sim_{j}$ $\mathbf{w}_{B}^{* *} \mathbf{x}_{-B}$.

In other words, the decision maker's preferences satisfy coordinate independence unconditionally with respect to $\mathscr{A}$-events and conditionally with respect to $\mathscr{B}$-events given any element of $\mathscr{A}$. $\mathscr{A}$-independence and conditional $\mathscr{B}$-independence are similar to the time- 0 and time- 1 substitution axioms of Kreps and Porteus (1979), adapted to a setting of uncertainty rather than risk and stripped of their temporal interpretation. (See also Segal 1987, 1989 and Grant et al. 1998 for related ideas.)

The intuition for $\mathscr{A}$-independence is similar to the intuition for restrictions of independence in other axiomatic models of uncertainty aversion (e.g., comonotonic independence, certainty independence, and weak certainty independence), namely, that it requires the direction of preference between two acts to be preserved only under common payoff substitutions or mixtures that do not hedge the ambiguity of one act more than the other. Coordinate independence requires that when two acts agree in some states, it does not matter how they agree there: if the agreeing payoffs are replaced by some other agreeing payoffs, the direction of preference must remain the same. This is violated in Ellsberg's (1961) experiments because the agreeing payoffs are changed precisely so as to hedge the ambiguity in one of the acts, while injecting ambiguity into the alternative act. For example, in the two-urn problem of Table 2, if the agreeing payoffs of $\mathbf{a}$ and $\mathbf{b}$ in the first and last rows are replaced by agreeing payoffs of identical magnitudes but with opposite signs, then $\mathbf{a}$ becomes $\mathbf{d}$ and $\mathbf{b}$ becomes $\mathbf{c}$, so coordinate independence requires $\mathbf{b} \succcurlyeq \mathbf{a}$ if and only if $\mathbf{c} \succcurlyeq \mathbf{d}$, which is violated by most subjects because this substitution eliminates the ambiguity in the expected payoff of a while adding ambiguity to the expected payoff of $\mathbf{b}$. $\mathscr{A}$-independence fails to bite on this problem because $\mathbf{a}$ and $\mathbf{b}$ do not agree on any $\mathscr{A}$-event in its entirety, so the typical preference pattern is not disallowed. In general, the only substitutions that must preserve preferences under $\mathscr{A}$-independence are those that do not change the agreeing payoffs of the acts in any ambiguous event where there is disagreement in some of the other states, thus preventing changes in the difference between their "uncertainty profiles."

Similarly, in the single-urn problem of Table 3, if the agreeing payoffs of 0 of $\mathbf{a}$ and $\mathbf{b}$ in the last row are replaced by agreeing payoffs of 100, then $\mathbf{a}$ becomes $\mathbf{e}$ and $\mathbf{b}$ becomes $\mathbf{d}$, so coordinate independence requires $\mathbf{a} \succcurlyeq \mathbf{b}$ if and only if $\mathbf{e} \succcurlyeq \mathbf{d}$, which is violated by most subjects because this substitution eliminates the ambiguity in the expected payoff of $\mathbf{b}$ while adding ambiguity to the expected payoff of a. Again, $\mathscr{A}$-independence fails to bite because a and $\mathbf{b}$ (and/or $\mathbf{c}$ ) do not agree on any $\mathscr{A}$-event in its entirety, as the refinement of Table 3 indicates; see Table 5.

Meanwhile, the intuition for conditional $\mathscr{B}$-independence is that conditioning on a singleton in $\mathscr{A}$ resolves all of the ambiguity in the situation, leaving only the risk of the $\mathscr{B}$-events to be considered, for which the decision maker is assumed to have (statedependent) SEU preferences.

Table 5 Payoff Matrix for the Refined Single-Urn Paradox

\begin{tabular}{lrrrrrr}
\hline & a & b & c & d & e & $\mathbf{f}$ \\
\hline$A_{1} B_{1}$ (Red first then yellow) & 100 & 0 & 0 & 0 & 100 & 100 \\
$A_{1} B_{2}$ (Yellow first) & 0 & 100 & 0 & 100 & 0 & 100 \\
$A_{2} B_{1}$ (Red first then black) & 100 & 0 & 0 & 0 & 100 & 100 \\
$A_{2} B_{2}$ (Black first) & 0 & 0 & 100 & 100 & 100 & 0 \\
\hline
\end{tabular}


Based on these axioms, this paper's main result is the following:

Theorem 1. (a) Axioms 1-3 hold if and only if $\succcurlyeq$ is represented by an observable, cardinal utility function of the composite-additive form:

$$
\text { Model I: } \quad U(\mathbf{w})=\sum_{j=1}^{J} u_{j}\left(\sum_{k=1}^{K} v_{j k}\left(w_{j k}\right)\right)
$$

where $\left\{u_{j}\right\}$ and $\left\{v_{j k}\right\}$ are strictly increasing twice-differentiable functions. $U$ is unique up to positive affine transformations, $\left\{v_{j k}\right\}$ are unique up to transformations of the form $\alpha_{j} v_{j k}+\beta_{j k}$, and $\left\{u_{j}\right\}$ are unique up to transformations of the form $\gamma u_{j}+\eta_{j}$ given $\left\{v_{j k}\right\}$, where $\left\{\beta_{j k}\right\}$ and $\left\{\eta_{j}\right\}$ are arbitrary constants and $\left\{\alpha_{j}\right\}$ and $\gamma$ are arbitrary positive constants.

(b) The corresponding risk-neutral probabilities satisfy

$$
\pi_{j k} \propto u_{j}^{\prime}\left(v_{j}(\mathbf{w})\right) v_{j k}^{\prime}\left(w_{j k}\right), \quad \text { where } v_{j}(\mathbf{w}) \equiv \sum_{k=1}^{K} v_{j k}\left(w_{j k}\right)
$$

(c) The local risk-aversion matrix $\mathbf{R}(\mathbf{w})$ is the sum of a diagonal matrix and a block-diagonal matrix, with $r_{j k, m n}(\mathbf{w})=0$ if $j \neq m$, and

$$
r_{j k, j n}(\mathbf{w})=-\frac{u_{j}^{\prime \prime}\left(v_{j}(\mathbf{w})\right)}{u_{j}^{\prime}\left(v_{j}(\mathbf{w})\right)} v_{j n}^{\prime}\left(w_{j n}\right)-\frac{v_{j k}^{\prime \prime}\left(w_{j k}\right)}{v_{j k}^{\prime}\left(w_{j k}\right)} 1_{k n} .
$$

(d) Let $\mathbf{s}$ and $\mathbf{t}$ denote J-and JK-vectors uniquely defined by

$$
s_{j}=-\frac{u_{j}^{\prime \prime}\left(v_{j}(\mathbf{w})\right)}{u_{j}^{\prime}\left(v_{j}(\mathbf{w})\right)} \sum_{k=1}^{K} v_{j k}^{\prime}\left(w_{j k}\right), \quad t_{j k}=-\frac{v_{j k}^{\prime \prime}\left(w_{j k}\right)}{v_{j k}^{\prime}\left(w_{j k}\right)} .
$$

Let $\pi_{j}=\sum_{k=1}^{K} \pi_{j k}$ and $\pi_{k \mid j}=\pi_{j k} / \pi_{j}$ denote the induced marginal and conditional risk-neutral probabilities, and let $\overline{\mathbf{z}}$ be defined as the J-vector, whose jth element is $\bar{z}_{j}=$ $\sum_{k=1}^{K} \pi_{k \mid j} z_{j k}$, i.e., the conditional risk-neutral expectation of $\mathbf{z}$ given event $A_{j}$. In these terms, the (total) risk premium for a neutral asset $\mathbf{z}$ is approximated by

$$
\begin{aligned}
b(\mathbf{z} ; \mathbf{w}) & \approx \frac{1}{2} \sum_{j=1}^{J} \pi_{j} s_{j} \bar{z}_{j}^{2}+\frac{1}{2} \sum_{j=1}^{J} \sum_{k=1}^{K} \pi_{j k} t_{j k} z_{j k}^{2} \\
& =\frac{1}{2} E_{\pi}\left[\mathbf{s} \overline{\mathbf{z}}^{2}\right]+\frac{1}{2} E_{\pi}\left[\mathbf{t z}^{2}\right],
\end{aligned}
$$

where the first term on the right-hand side will be henceforth called an uncertainty premium and the second term will be called a pure risk premium.

(e) For every $\mathscr{B}$-event $B$ and sufficiently small $\Delta$, the uncertainty premium of a $B$-spread of size $\Delta$ is zero to a second-order approximation.

(f) The decision maker is locally uncertainty averse if $\mathbf{s} \geq \mathbf{0}, \mathbf{s} \neq \mathbf{0}$, and (globally) uncertainty averse if $u_{j}$ is concave for every $j$ and strictly concave for at least one $j$.
Here, $v_{j k}$ is a first-order evaluation function for money in state $A_{j} B_{k}$, while $u_{j}$ is a second-order evaluation function whose argument is the total first-order value of the payoff profile in event $A_{j}$. By analogy with the familar Pratt-Arrow measure, the term $t_{j k}=$ $-v_{j k}^{\prime \prime} / v_{j k}^{\prime}$ in the total risk premium formula of part (d) is an observable local measure of aversion to pure risk in state $A_{j} B_{k}$, while the term $s_{j}=-\left(u_{j}^{\prime \prime} / u_{j}^{\prime}\right)\left(\sum_{k} v_{j k}^{\prime}\right)$ is an observable local measure of aversion to the uncertainty (ambiguity) surrounding event $A_{j}$. (The term $u_{j}^{\prime \prime} / u_{j}^{\prime}$ by itself has units of $v^{-1}$. When multiplied by $\sum_{k} v_{j k}^{\prime}$, which is the aggregate marginal value for money in event $A_{j}$, the resulting units for $s_{j}$ are dollars $^{-1}$, the same as for $t_{j k}$.) By construction, an $A$-spread and its corresponding $B$-spread always have the same pure risk premium, but the uncertainty premium of the latter is zero, while that of the former is strictly positive for an uncertainty averse decision maker.

In Model I, the first- and second-order evaluation functions are state dependent and there is no separation of probability from utility. A state-independent representation that separates probability from utility - at least in an "as if" sense-can be obtained by imposing stronger conditions of trade-off consistency, which are a cross-state form of independence (Wakker 1989, Tversky and Wakker 1993). Henceforth, let $a \mathbf{w}_{-j k}$ denote the act that yields $a$ in state $A_{j} B_{k}$ and which agrees with $\mathbf{w}$ in every other state. Let $a \mathbf{w}_{-j}$ denote the act that yields $a$ in event $A_{j}$ and which agrees with $\mathbf{w}$ in every other state. Define an act $\mathbf{w}$ to be $\mathscr{A}$-constant if its payoffs are constant within each event $A_{j}$ (i.e., if its payoffs depend only on which element of $\mathscr{A}$ occurs).

Axiom $3^{*}$ (Conditional $\mathscr{B}$-Trade-off ConsisTENCY). For any acts $\mathbf{w}, \mathbf{x}, \mathbf{y}, \mathbf{z}$, and any $j, j^{*} \in\{1, \ldots, J\}$ and $k, k^{*} \in\{1, \ldots, K\}$, if $a \mathbf{w}_{-j k} \preccurlyeq_{j} b \mathbf{x}_{-j k}$ and $c \mathbf{w}_{-j k} \succcurlyeq_{j}$ $d \mathbf{x}_{-j k}$ and $a \mathbf{y}_{-j^{*} k^{*}} \succcurlyeq_{j^{*}} b \mathbf{z}_{-j^{*} k^{*}}$, then $c \mathbf{y}_{-j^{*} k^{*}} \succcurlyeq_{j^{*}} d \mathbf{z}_{-j^{*} k^{*}}$.

Axiom 4 (Trade-off Consistency for $\mathscr{A}$-ConSTANT ACTS). For any $\mathfrak{A}$-constant acts $\mathbf{w}, \mathbf{x}, \mathbf{y}, \mathbf{z}$, and any $j, j^{*} \in\{1, \ldots, J\}$, if $a \mathbf{w}_{-j} \preccurlyeq b \mathbf{x}_{-j}$ and $c \mathbf{w}_{-j} \succcurlyeq_{j} d \mathbf{x}_{-j}$ and $a \mathbf{y}_{-j^{*}} \succcurlyeq b \mathbf{z}_{-j^{*}}$, then $c \mathbf{y}_{-j^{*}} \succcurlyeq d \mathbf{z}_{-j^{*}}$.

Theorem 2. Axioms 1, 2, 3*, and 4 are satisfied if and only if $\succcurlyeq$ is represented by a utility function of the form

$$
\text { Model II: } \quad U(\mathbf{w})=\sum_{j=1}^{J} p_{j} u\left(\sum_{k=1}^{K} q_{j k} v\left(w_{j k}\right)\right),
$$

where $\mathbf{p}$ is a unique marginal probability distribution on $\mathscr{A}, \mathbf{q}_{j}$ is a unique conditional probability distribution on $\mathscr{B}$ given $A_{j}, v$ is a strictly increasing state-independent first-order Bernoulli utility function unique up to positive affine transformations, and $u$ is a strictly increasing stateindependent second-order Bernoulli utility function unique up to positive affine transformations given $v$. 
Thus, under Model II the decision maker behaves as though she assigns probability $p_{j} q_{j k}$ to state $A_{j} B_{k}$, and she bets on $\mathscr{A}$-events as though her utility function was $u(v(w))$. Meanwhile, conditional on any element of $\mathscr{A}$ (or unconditionally if $\mathscr{A}$ and $\mathscr{B}$ are probabilistically independent so that $q_{j k}$ does not depend on $j$ ), she bets on $\mathscr{B}$-events as though her utility function for money was $v(w)$. (This result is very similar to Ergin and Gul's (2004) Theorem 3, except that $\mathscr{A}$ and $\mathscr{B}$ are not required to be probabilistically independent.) The usual caveats apply here, namely, that the uniqueness of the probabilities depends on the conventional but unverifiable assumption that $u$ and $v$ do not have state-dependent scale factors, and the set of acts that are $\mathscr{A}$-constant may not be apparent to an observer if the decision maker has unknown prior stakes in the $\mathscr{B}$-events.

If $u$ is strictly concave in Model II, the decision maker is uniformly more risk averse with respect to bets on $\mathscr{A}$-events than to bets on $\mathscr{B}$-events, despite appearing to have equally precise probabilities for both: concavity of $v$ models her aversion to risk, while concavity of $u$ models her aversion to the uncertainty surrounding the $\mathfrak{A}$-events. Similarly, convexity of either function would represent attraction to risk or uncertainty. In the illustrative utility functions (2) and (3) that rationalize Ellsberg's (1961) two-urn and single-urn paradoxes, $v$ is linear and $u$ is concave, hence, the decision maker is risk neutral but uncertainty averse.

These models could, in principle, be extended to threefold partitions, fourfold partitions, etc., all having different degrees of uncertainty, via deeper nestings of the independence axiom, although a twofold partition suffices to demonstrate distinct attitudes toward risk and uncertainty.

\section{Conclusions}

This paper has presented a simple model of uncertainty-averse non-EU preferences that does not involve kinked indifference curves, objective randomization, probabilistically sophisticated benchmarks, nor imaginary acts; and, in particular, it has been shown that uncertainty aversion can be detected and measured as an "uncertainty premium" even in the presence of state-dependent utility. The decision maker may, for whatever reason, display different degrees of risk aversion toward two (or more) different classes of events, which leads to a simple behavioral definition of aversion to uncertainty that does not refer to either objective or subjective probabilities; namely, the decision maker is uncertainty averse if she is systematically more risk averse toward certain kinds of "spread" bets on events she regards as more ambiguous than toward corresponding bets on logically independent events she regards as less ambiguous. A twofold partition of events has been assumed for simplicity in obtaining closed-form results, but the same approach could be applied more generally. A decision maker may be uncertainty averse by this standard and yet conform (approximately) to subjective EU theory with respect to acts that are pegged to the same source of risk or uncertainty. The unique separation of probabilities and utilities is not essential in this framework, although it can be formally achieved by imposing versions of the usual trade-off consistency conditions. However, as has been shown elsewhere (Nau 2003), it is possible to model risk aversion and carry out decision analysis without separating probabilities from utilities, and there are compelling epistemic reasons for demonstrating that this can be done. The theory of choice under uncertainty outlined in this paper does not necessarily invalidate conventional decision-analytic methods of assessing risk tolerances; however, it suggests that those methods should perhaps be tailored to specific sources of uncertainty for uncertainty-averse decision makers.

\section{Acknowledgments}

This research was supported by the National Science Foundation under grant 98-09225 and by the Fuqua School of Business. The author is grateful for the comments of participants at ISIPTA '01, RUD 2002, and JSM 2002, the department editor, and two anonymous referees.

\section{References}

Anscombe, F., R. Aumann. 1963. A definition of subjective probability. Ann. Math. Statist. 34 199-205.

Arrow, K. J. 1964. The role of securities in the optimal allocation of risk-bearing. Quart. J. Econom. 31 91-96.

Aumann, R. 1971. Letter to L. J. Savage. Reprinted in J. Drèze. 1987. Essays on Economic Decisions under Uncertainty. Cambridge University Press, London, UK

Chew, S. H., J. Sagi. 2003. Small worlds: Modeling attitudes toward sources of uncertainty. Working paper, University of California, Berkeley, CA.

Debreu, G. 1959. Theory of Value. Cowles Foundation, New Haven, CT.

Debreu, G. 1960. Topological methods in cardinal utility theory. K. J. Arrow, S. Karlin, P. Suppes, eds. Mathematical Methods in the Social Sciences. Stanford University Press, Stanford, CA, $16-26$.

Ellsberg, D. 1961. Risk, ambiguity and the savage axioms. Quart. J. Econom. 75 643-669.

Epstein, L. 1999. A definition of uncertainty aversion. Rev. Econom. Stud. 66 579-608.

Epstein, L., J.-K. Zhang. 2001. Subjective probabilities on subjectively unambiguous events. Econometrica 69 265-306.

Ergin, H., F. Gul. 2004. A subjective theory of compound lotteries. Working paper, Princeton University, Princeton, NJ.

Ghirardato, P., M. Marinacci. 2001. Risk, ambiguity, and the separation of utility and beliefs. Math. Oper. Res. 26 864-890. 
Ghirardato, P., M. Marinacci. 2002. Ambiguity made precise: A comparative foundation. J. Econom. Theory 102 251-289.

Gilboa, I., D. Schmeidler. 1989. Maxmin expected utility with a nonunique prior. J. Math. Econom. 18 251-289.

Grant, S., A. Kajii, B. Polak. 1998. Intrinsic preference for information. J. Econom. Theory 83 233-259.

Hirshleifer, J. 1965. Investment decision under uncertainty: Choicetheoretic approaches. Quart. J. Econom. 74 509-536.

Karni, E., P. Mongin. 2000. On the determination of subjective probability by choices. Management Sci. 46 233-248.

Karni, E., D. Schmeidler, K. Vind. 1983. On state dependent preferences and subjective probabilities. Econometrica 51 1021-1031.

Klibanoff, P., M. Marinacci, S. Sukerji. 2005. A smooth model of decision making under ambiguity. Econometrica 73 1849-1892.

Kreps, D., E. Porteus. 1979. Temporal Von Neumann-Morgenstern and induced preferences. J. Econom. Theory 20 89-109.

Maccheroni, F., M. Marinacci, A. Rustichini. 2004. Ambiguity aversion, malevolent nature, and the variational representation of preferences. Working paper, University of Turin.

Nau, R. F. 2001. De Finetti was right: Probability does not exist. Theory Decision 51 89-124.

Nau, R. F. 2003. A generalization of Pratt-Arrow measure to nonexpected-utility preferences and inseparable probabilities and utilities. Management Sci. 49(8) 1089-1104.
Pratt, J. W. 1964. Risk aversion in the small and in the large. Econometrica 32 122-136.

Savage, L. J. 1954. The Foundations of Statistics. Wiley, New York. (1972, 2nd ed., Dover, New York.)

Schervish, M. J., T. Seidenfeld, J. B. Kadane. 1990. State-dependent utilities. J. Amer. Statist. Assoc. 85 840-847.

Schmeidler, D. 1989. Subjective probability and expected utility without additivity. Econometrica 57 571-588.

Segal, U. 1987. The Ellsberg paradox and risk aversion: An anticipated utility approach. Internat. Econom. Rev. 28 175-202.

Segal, U. 1989. Two-stage lotteries without the reduction axiom. Econometrica 58 349-377.

Shafer, G. 1986. Savage revisited. Statist. Sci. 1 463-501.

Tversky, A., D. Kahneman. 1992. Advances in prospect theory: Cumulative representation of uncertainty. J. Risk Uncertainty 5 297-323.

Tversky, A., P. P. Wakker. 1993. An axiomatization of cumulative prospect theory. J. Risk Uncertainty 7 147-176.

Wakker, P. P. 1989. Additive Representations of Preferences: A New Foundation of Decision Analysis. Kluwer Academic Publishers, Dordrecht, The Netherlands.

Yaari, M. 1969. Some remarks on measures of risk aversion and their uses. J. Econom. Theory 1 315-329. 